\title{
Estimation at Completion in Civil Engineering Projects: Review of Regression and Soft Computing Models
}

\author{
Almahdi Mohamed Araba ${ }^{1}$, Musab Alhawat ${ }^{2}$, Solomon Sackey ${ }^{3}$, Mumtaz Ali ${ }^{4}, Z^{2 u b a i r ~ A h m e d ~ M e m o n ~}{ }^{5}$ \\ and Abdalrhman Milad6,* \\ ${ }^{1}$ Arcadis Design \& Consultancy, Heworth Green, York, United Kingdom; Almahdi.araba@arcadis.com \\ ${ }^{2}$ Faculty of Engineering and Informatics, University of Bradford, United Kingdom; malhawat@ bradford.ac.uk \\ ${ }^{3}$ Dept. of Civil Engineering, Kyungpook National University, Daegu, 41566, Korea; s.sackey123@knu.ac.kr \\ ${ }^{4}$ Deakin-SWU Joint Research Centre on Big Data, School of Information Technology, Deakin University, Victoria \\ 3125, Australia; mumtaz.ali@deakin.edu.au \\ ${ }^{5}$ Department of Engineering Management, College of Engineering, Prince Sultan University (PSU), Riyadh 11586, \\ Saudi Arabia; zamemon@psu.edu.sa \\ ${ }^{6}$ Dept. of Civil Engineering, Faculty of Engineering and Built Environment, Universiti Kebangsaan Malaysia, Selangor, \\ Malaysia; \\ * Correspondence: abdalrhmanmiladly@gmail.com
}

Manuscript received: 23-02-2021, revised: 14-08-2021, accepted: 16-08-2021.

\begin{abstract}
Construction projects are usually associated with several challenges owing to the varying process during the project lifetime. Hence, the final cost of any civil engineering project is influenced by many factors. There are numerous ways of determining the final cost of a project, however, the most essential approach is the Estimate at Completion (EAC) technique. This technique is mostly favored because it considers the probability of risks and project performance. Furthermore, EAC helps project managers in the definition and determination of the critical problems expected during the project period and the likely solutions toward these problems. In this review research, the basic empirical, regression and advanced soft computing methodologies adopted for the EAC computation, were surveyed and reported in detail. The review established on the base to recognize the modern advancement of the soft computing in computing the EAC with accurate, reliable and robust manner. The review was highlighted the main literature limitation, current status and possible future direction.
\end{abstract}

Keywords: Estimate at Completion; review; regression models; soft computing.

\section{Introduction}

Construction site is a risky environment and always has many problems during the execution period [1], [2]. Managing this sector effectively represents a specific challenge because of the construction nature that characterized by a changeable environment and complex techniques [3], [4]. As a result, construction firms obligate to monitor the project cost to predict the variations and take a proper decision [5], [6]. During planning process, the project managers focused on managing project budget at initial stage and neglect the impact of cost changes and information updates in other project phases [7]. This leads to improper cost control and prevent the prediction of future problems. The changing environment of construction industry produce frequently alterations in the project budget and this action is considered a critical aspect in cost management.

Earned value management (EVM) is a management tool that capable to control the project process within time, cost and scope [8], [9]. Through using EVM formula, project manager computes estimation at completion (EAC) by evaluating the costs of planned work. Computing of EAC helps the manger to measure the difference between the actual and planned cost and identify the potential problem [10].

Using of EVM tool has one drawback that is the availability of many methods for EAC calculation. As mention in published literatures, there are eight formulas have been used for this purpose [11]. To calculate EAC, the mangers must select the appropriate formula depending on his experience [12]. There are many factors are affecting the project cost and every project has special characteristics that may not exist in another project. This makes the efficiency of EAC formulas is difference between construction projects [7].

Traditional deterministic formula is considered an expensive and impractical method to estimate EAC in construction site due to the complexity and uncertainty of construction process [13], [14]. Developing an approximate model has the ability to deal with such circumstances represents a practical method [15]. The approximate model is transparent and depends on predicting new data by using historical information. The dataset is adjustable, and the input data can be changed while the model remains constant. The phenomenon of 
detecting new data was deriving from human brain. Thus, artificial intelligence (AI) and soft computing can be used to develop methods have the ability to mimic human brain system [16], [17]. Soft computing refers to computer system has the ability to deal with complex system using models such as Artificial Neural Network (ANN), fuzzy logic, extreme learning machine [18], Support Vector Machine and others [19]. In the research domain of project management, the implementations of AI models have been progress massively. For instance, A Sugeno fuzzy model was suggested and investigated for project planning and scheduling [20]. The authors attempted to tackle the associated uncertainties with the project planning and come up with a reliable software for the project management. Adaptive neuro-fuzzy inference system (ANFIS) model was tested for project evaluation based on key management indicators, planning, costs, data quality, human resources, effectiveness and logistic, the research was evidenced the capacity of the decision-making support tool [21]. The development of several version of AI models including fussy logic, neural network, K-means clustering for cash flows of project management was conducted and research results approved the potential of the proposed models [22]. Using of soft computing in EAC estimation represents a promising way to handle with such problems.

\section{Estimation at completion concept}

In construction management, Earned Value Management can be defined as the management method used to measure performance requirements in terms of cost and time for the given project [23]. Project manager used EVM technique to monitor project situation and evaluate project. The major elements of EVM are: Budgeted Cost of Work Scheduled (BCWS), Budgeted Cost of Work Performed (BCWP) and Actual Cost of Work Performed (ACWP). In EVM technique, project manager relies on EAC to forecast the project cost [24]. The importance of EAC is highlighted because of the ability of EAC concept in estimation the total cost with the hypothesis that past project tasks can affect the future outcome of construction project. Estimation at completion is calculated by many formulas depending on the data supplied by the contractor in monthly report. Periodic report must supply by the owner to contractor containing all sufficient data in construction project. These data included daily summary of man-hours, material and equipment in construction site. By these information in the progress report, EAC evaluated by calculating the elements: BCWS, BCWP and ACWP [11]. Various indexbased methods have been used to estimate EAC [7], [12], [25].

where BAC refers to budget at completion and $W_{1}$ is the complementary weight. The effectiveness of these methods relied on the system type and project phase. Thus, the accuracy of these methods is differing among the construction projects. This drawback of traditional methods leads to develop more accurate prediction model.

Despite the importance of cost control in construction projects, it is a difficult and time-wasting process due to the need to individually consider all the factors that can affect the project cost at each project stage [7]. EAC remains an important indicator for cost control performance [26]-[28]; however, EAC must be accurately calculated to ensure appropriate identification of the problems and the necessary solutions. Project managers normally rely on Earned Value Management (EVM) for EAC calculation [29]-[31] as this approach integrates both the project cost, scope and schedule metrics into a single measurement system for the proper analysis and measurement of the actual project actual against its baseline, as well as to accurate determine the cost and duration of a project at completion [32].

It has been stated that the EVM is gaining broader acceptance owing to the increase in the acknowledgement of its capability of reducing the problems of EVM as well as improving utilities [33]. However, traditional EVM methods have some limitations. For example, the index-based EVM methods have the limitation of using only the previous information and performance index to calculate the remaining project budget [34]. Furthermore, the project costs provided by these models at the early stages of project life are unreliable as they are made based on few EVM data [31]. Even though the traditional EVM methods can provide accurate predictions when employed on certain special projects, they are often erroneous in most cases and can lead to an industrial situation of being confused about the right prediction approach that will be appropriate for a project. The EVM is also suffering from the issue of revisions being conducted manually at each stage of the project; this makes EVM a tedious and time-waiting approach. Consequently, it is critical to computerize engineering management process if EVM is to be deployed effectively for project cost control. In most construction companies, computer systems are usually used for the analysis of project budgets at the initial stages because they are reasonably powerful in such analysis. The computer system may not appropriately respond to changes at each project stage or predict construction project EAC using the EVM method. Hence, the development of using artificial intelligence (AI) models to solve this problem is extensively attractive for the new era of research trend for project engineering scholars. Over the literature, the applications of machine learning models have been demonstrated a remarkable progress on simulating the EAC process as per the reported systematic literature of the Scopus database presented in Figure 1. 

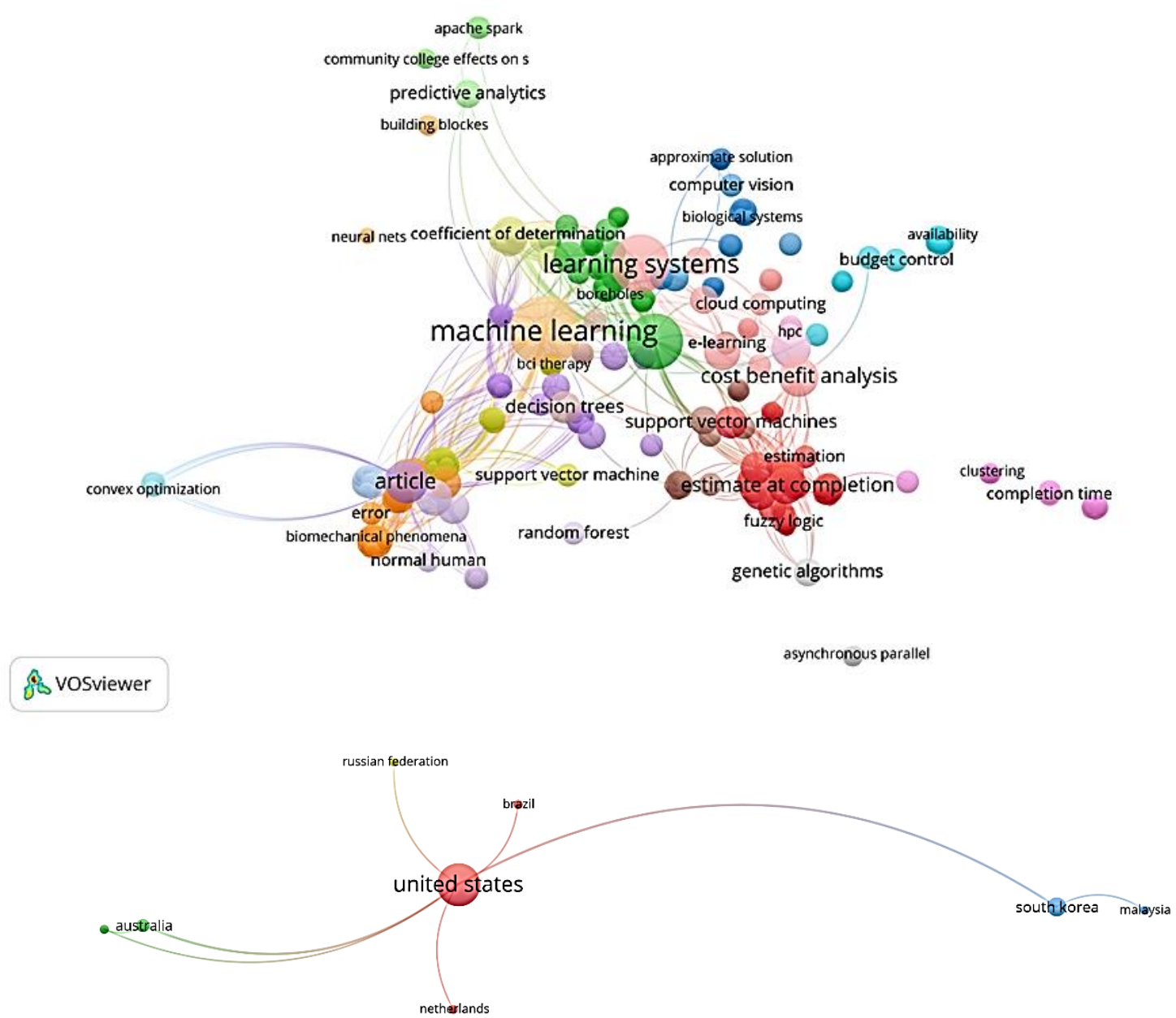

Figure 1. The dataset abstracted from the Scopus database on the EAC simulation using machine learning models and the countries adopted research on this topic.

\section{Indexed Formulation for EAC}

The cost management data which the contractor provided in the Cost Performance Report or the Cost/Schedule Status Report can be used to formulate the EAC [35]. The reviewed studies presumed the reliability of data presented in these reports. Data reliability is dependent on the degree a contractor adheres to an internal control system which involves the budgeting, scheduling, and analysis of contractual effort.

All formulas for EAC estimation depend on the integration of numerous data parameters contained in the cost management report. These data parameters include the Actual Cost (AC) of the performed work at the Actual Time (AT) and estimated cost of the remaining work. In addition, the difference between earned value (EV) and the budget at completion (BAC) in which adjusted by the performance index (PI). The formulas for EAC estimation are grouped into artificial intelligence modeling, index, and regression methodologies. Equation (1) represents the generic index-based formula.

$$
E A C=A C+(B A C-E V) / \mathrm{PI}
$$

The adjustment of the PI is mainly based on the assumption that the past cost and schedule performance of the project reflects the future performance. Figure 2 presented the addressed the EAC problem. There are four groups of performance indices including Cost Performance Index (CPI), Schedule Performance Index (SPI), Schedule Cost Index (SCI), and Composite Index (CI). These indices can either be collected monthly, as an average, or cumulatively, while the following conventions are adopted for the labelling: $\mathrm{CPI}_{\mathrm{m}}=\mathrm{CPI}$ based on the most recent month; $\mathrm{CPI}_{\mathrm{c}}=$ cumulative $\mathrm{CPI} ; \mathrm{CPI}_{\mathrm{x}}=\mathrm{CPI}$ divided by $x$ number of months, starting from the recent to the later month. For instance, $\mathrm{CPI}_{3}$ is presented 3 months average pf $\mathrm{CPI}$ calculated from the current month and the immediate past 2 months. The same conventions are used for SPI and SCI; for instance, $S_{\text {PI }}$ represents a six months average SPI calculated from the current month and the immediate past 5 months. 


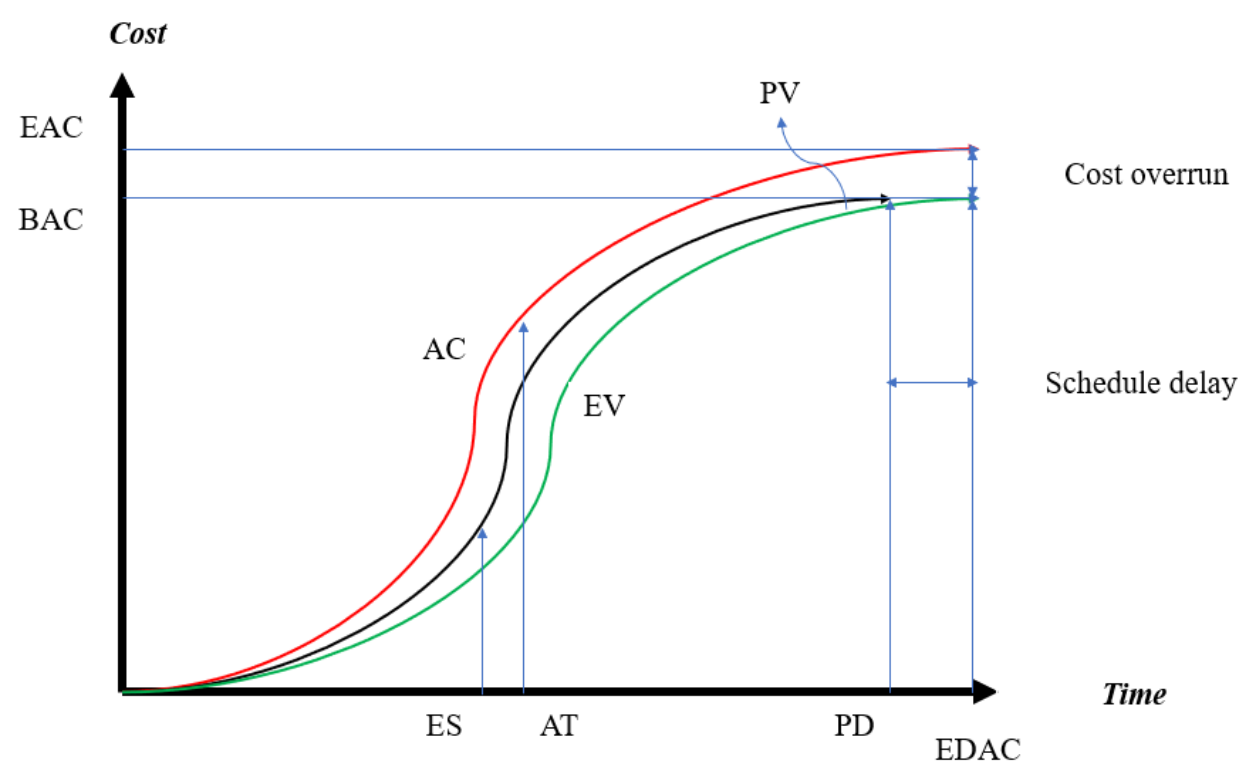

Figure 2. EAC graph as per the cost and time of any construction project.

The $2^{\text {nd }}$ and $3^{\text {rd }}$ groups of EAC formulas are called "regression" and "other." Either linear or nonlinear regression analysis is used to derive the regression-based EAC formulas. Non-linear regression analysis, in this paper, is defined in this paper as nonlinear relationship analysis irrespective of the possibility of transforming it into a linear relationship 3. In most cases, the ACWP is often the dependent variable while BCWP or time is the independent variable. The "other" category comprised of formulas that fall outside of the first two categories, such as the heuristics-based formulas.

Apparently, there are several formulas for the estimation of EAC and this has placed the analyst in the position of deciding the right formula to be used. The Performance Analyzer is a well-known analysis software which gives the user an opportunity to select from a range of formulas. However, there is no guideline on how to select the best or accurate formula. This issue will be addressed in the remaining parts of this work by reviewing the previous studies conducted on EAC in the last sixteen years.

\section{Literature review}

\subsection{Empirical Formulation for EAC}

The empirical formulations for EAC describe a "new" modeling methodology which generally does not involve statistical or sophisticated heuristic technique but cannot be fully described as comparative analysis [36].

Index-based methods: A proposal for 4 empirical methods based on the composite index weights has been made [37]-[40]. The formulation of these methods depends on the subjectively assigned index weights. These studies believe that the information content of SPI is eventually lost since it is driven to unity at contract completion. As the project nears completion, the weight assigned to the SPI tend to decrease to zero. Haydon, in his $5^{\text {th }}$ study, relied on EACs estimated via several index-based formulas to derive a point estimate [41].

Jakowski [37] presented a complex heuristic approach for composite index weights determination of Navy Aviation Systems Command. The method first used CPIc until significant decreases are noted in the latest monthly-based CPIs before using an "optimally weighted" composite index which is considered to be the exact weight that resulted in the least historical standard deviation in the composite index. CPIc is once again used when the project nears $60 \%$ completion. Despite the unavailability of the original heuristic report from Jakowski, it has been described by [42].

Lollar [38] suggested the definition of the weights for cumulative SPI \& CPI as the contribution of the percentage variations in the cost \& schedule values to their total. This definition was later adopted by Blythe (1982) and Cryer (1986) in their comparative studies [43], [44]. However, it performed poorly against the other formulas.

A method proposed by Parker (Defense Logistics Agency, 1980) involves the computation of a range of composite indices by varying the weights in the range of 0 to 1 with increments of 0.1 [39]. Based on the conditions of the project, the analyst will decide the most appropriate composite index to be used.

With a suitable variable transformation, the general linear regression model can be applicable to inherently linear models. For instance, nonlinear cumulative cost growth patterns can be transformed into linear forms prior to their estimation using ordinary least squares. 
Composite index weights determination based on the percentage completion has been proposed by Totaro (Defense Logistics Agency 1987) [40]. The analyst assigns the starting weights for the SPI and CPI after considering the characteristics of the program, such as the predetermined manpower loading by the project executor.

Haydon \& Riether presented a way of determining point estimate from EACs calculated from a range of formulas [41]. First, index-based formulas are used to compute a range of EACs [42] before expanding the range by $2.5 \%$. The point estimate for the EAC was determined as the median of the expanded range. Upon the analysis of 21 completed or nearly completed projects handled by the Navy (15 development and 6 production projects), contractors with EAC value less than this point estimate are said to have a more accurate forecast most times. Then, the study provided a sample procedural worksheet and a numerical example.

\subsection{Regression-based Model for EAC Computation}

Regression-based methods: Three non-comparative methods on curvilinear cumulative cost growth profile modelling on defense contracts using regression analysis has been proposed. The proposed methods are complicated and well documented but require regression analysis skills; hence, they are difficult to implement. The forecasting of EAC using time series analysis has been proposed by Sincavage in 1974 [45]. The "Time Series Analysis for Army Internal Systems Management" (TSARISM) is a computer-based model which uses either moving average, autoregressive or a combination of both methods. The development of this model demand data collection over several months as it is sensitive to autocorrelation (a statistical problem). As such, the model is only useful at the later stages of a project. According to the author of this model, the original documentation is no longer available.

Olsen, et al [46] described the time-series prediction method used by the B-1 System Program Office wherein the EAC prediction was done using the "GETSA", a computer program that was put together by General Electric and managed by the B-1 SPO. A brief description of other techniques such as exponential smoothing and regression analysis was also provided. A numerical example was also provided.

An alternative approach to the development of non-linear regression-based model coefficients has been described by Busse (Air Command and Staff College, 1977) [47]. Although this description made no form of comparison with the Karsch model [48], a numerical instance was provided based on Karsch data. The comparison of the Busse and Karsch results at certain stages of project completion showed the EACs generated by the Karsch model to be more accurate [47].

The fitting of data from development programs into a normalized S-curve using nonlinear regression analysis has been proposed by Weida (Air Force Academy, 1977) [49]. After the adjustment of the data for statistical and inflation problems, Weida stated that the S-curve described the cumulative cost growth on all the examined 22 development programs. The normalized S-curve can then be employed for both predictive and comparative tasks. The study provided a numerical example. Despite the complicated nature of Weida's technique, it is compelling and deserves serious consideration.

The use of a time-series forecasting method called "Adaptive Forecasting" has been proposed by Chacko (Defense Systems Management College, 1981). This method requires 5 months of data before making an accurate EAC estimate [50]. The adaptive prediction models are subject to changes upon the availability of the data for each month. Hence, the models are best suited for short-term EAC prediction.

Watkins [51] proposed EAC prediction using linear regression analysis (LRA) and an adaptive version of the Rayleigh-Norden model. In this study, the model was used for LRA of ACWP against time based on quarterly data gathered from 3 contracts submitted to C/SSRs after data adjustment for inflation but without autocorrelation-related adjustment.

The calculation of EAC using a Bayesian probability theory has been proposed by El-Sabban (Army Aviation Systems Command, 1973) [52]. İn this method, assumptions were made at the initial phase of the project for a mean, a normal probability distribution, and a variance. Then, the Bayes's formula was used to reverse the "previous probability distribution" of the EAC owing to the availability of the current ACWP data. This model is more useful at the initial phase of a project since it is not dependent on a long history of performance data. Although the accuracy of the method has been challenged, it is still well presented [53] with the provision of an example.

Holeman [54] proposed EAC development using a "performance factor" that was determined based on subjective decision as a "product improved method". The performance factor is used as a performance index and includes a linear combination of several variables. İt is the duty of the analyst to determine the relative contribution of each variable. Holeman also suggested the subjective determination and evaluation of a range of EACs using simulation. The method presented a numerical example.

There are several limitations of the traditional EVM index-based approaches for EAC estimation of ongoing projects and these limitations are inherent with both early-stage unreliability and the belief that the best information can be sourced from the past EVM data. TO address these limitations, a modified index-based 
formula for CEAC estimation was proposed for the prediction of the expected cost for the remaining part of a project. This method employs the Gompertz growth model through a nonlinear regression curve fitting [30]. Determination of cost performance in the proposed method depends on schedule progress owing to the integration of its equation with an Earned Schedule-based factor that stipulates the expected period of completing a project. Hence, the model is ideal for both early, mid, and late-stage estimates compared to the earlier mentioned 4 index-based formulae. The method comes as a practical tool to the project managers when incorporating the progress status into CEAC computation task. İt also extended EVM studies by providing a better understanding of the relationship between cost and schedule factors.

A new regression-based nonlinear CEAC method which is a combination of a growth model and earned schedule (ES) concepts have been proposed to improve the accuracy of the early prediction of the final project cost at completion [32]. This method provides the CEAC for projects at the early and mid-completion stages. Hence, the research is formulated based on 3 major objectives; (i) to develop a new formula which is based on the combination of the ES method with four different growth models (Logistic, Gompertz, Bass, and Weibull); (ii) to validate the new method by applying it on 9 past projects; and (iii) to select the best-performing growth model based on their statistical validity test, as well as the comparison of the accuracies of their CEAC estimation. The comparison of the 4 growth models in terms of their CEAC error showed the Gompertz modelbased CEAC formula to provide a better fitting and generate more accurate final cost estimates compared to the estimated computed by the index-based method and the 3 other models. Theoretically, the proposed method contributed to the EAC study by the combination of EV metrics with regression-based studies. It also has a practical implication of using an accurate and viable forecasting method which considered schedule impact as a factor that determines the cost behavior.

\subsection{Soft Computing Models for EAC}

Several elements play essential roles in the construction of project feasibility studies; such elements include the basis of project evaluation, conceptual cost estimates, engineering design, cost management, and cost budgeting. In the practical perspective, construction cost estimates are generally reliant on the intuitive experience of the expert. To raise the accuracy of conceptual cost estimates, there is a need to develop and employ scientific methods during project design and planning. The improvement of the accuracy of cost estimates based on a new evolutionary fuzzy neural network has been proposed [55]. The study combined the advantages of genetic algorithms, neural networks, and fuzzy logic into one intelligent predictive model. This intelligent predictive model is highly useful in the identification of optimal solutions for complex problems. The structure of the adaptive neuro fuzzy inference system (ANFIS) was displayed in Figure 3. The authors concluded the study by providing two types of estimators which can be used to make a precise estimate of conceptual construction cost in the early phase of projects.

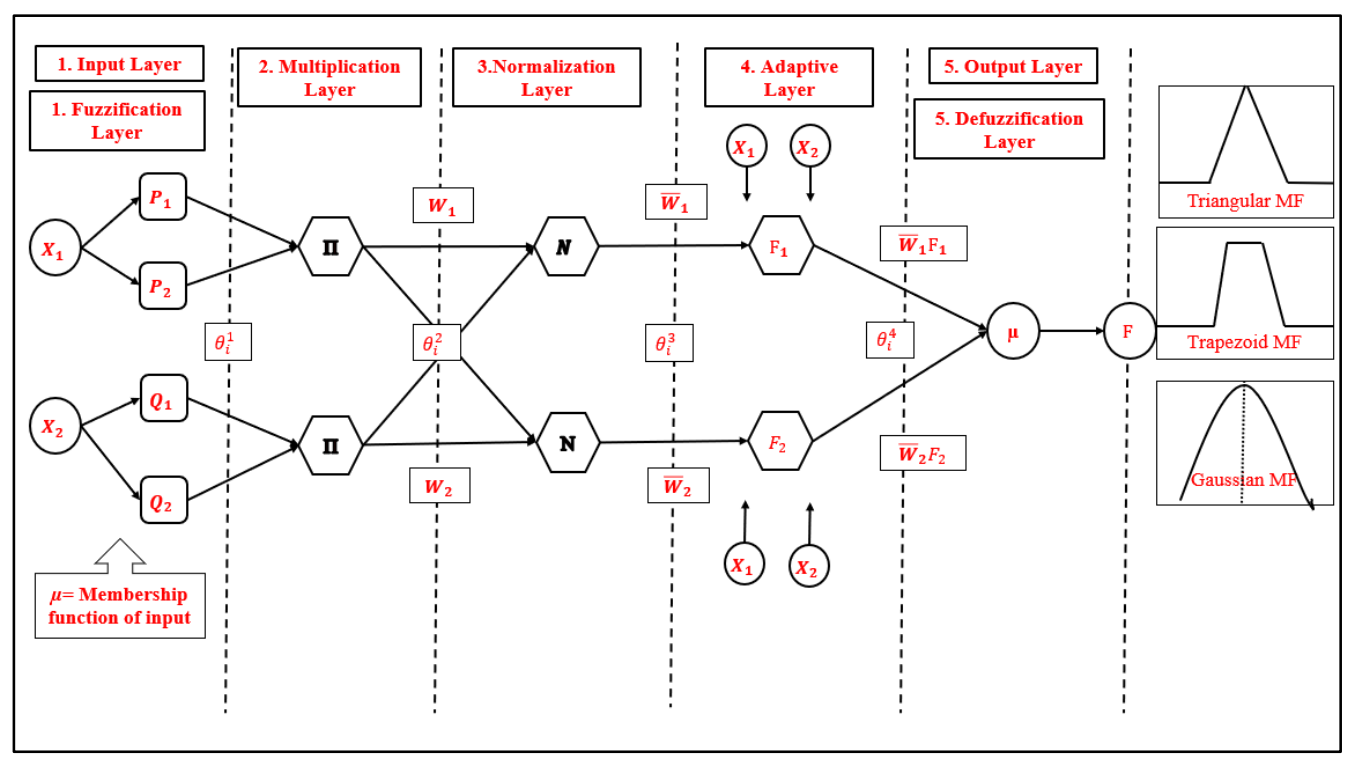

Figure 3. The structure of the ANFIS model.

Due to the challenging nature of construction projects, construction decision makers have been compelled to successfully work in an environment characterized by frequent complexity and full of uncertainties. Being that numerous decisions are intuitively made based on limited data, two factors are mainly involved in making a successful decision; these are the experience of the concerned expert(s) and the quality of accumulated 
knowledge from past experiences. However, the value and accuracy of knowledge can be influenced by several factors. The evolutionary fuzzy support vector machine inference model and fast messy genetic algorithm (fmGA) were developed as an alternative method towards utilizing and retaining knowledge gained from experience [56] (Figure 4). The study defined 2 actual and simulated construction management problems and showed the proposed hybrid intelligent model as an effective method of solving several construction-related problems.

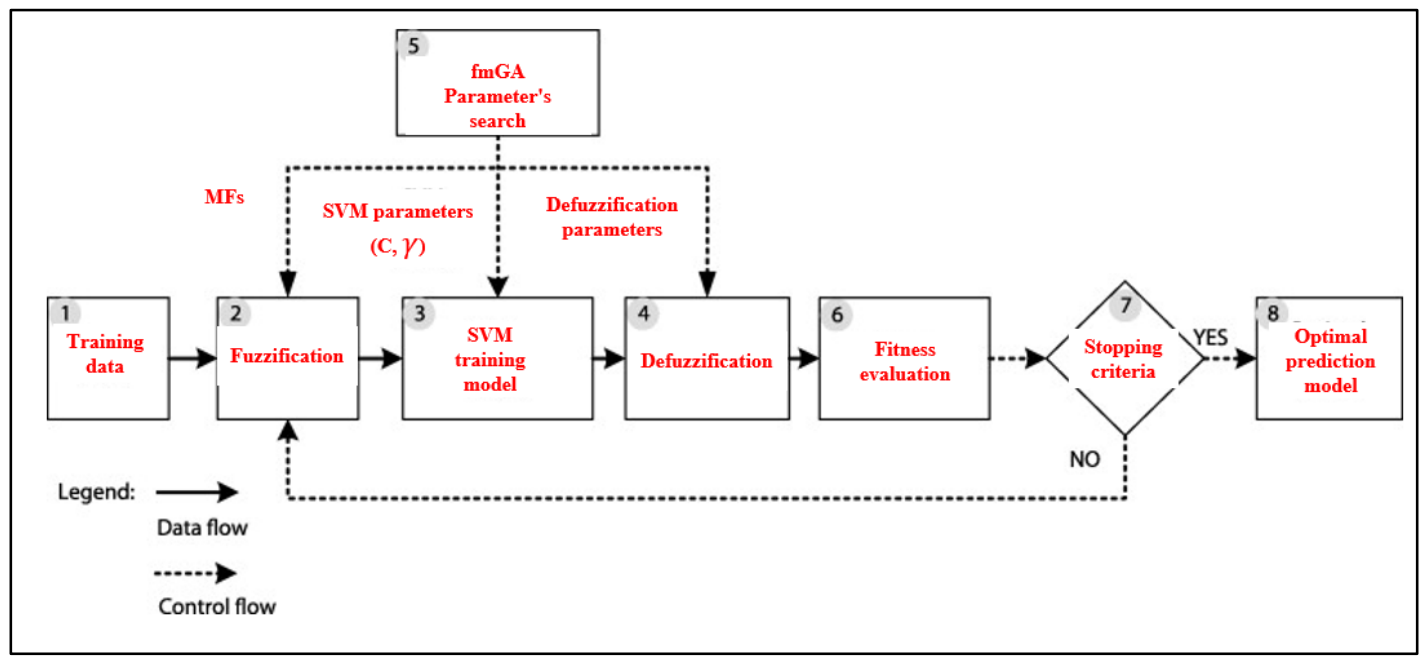

Figure 4. The adopted evolutionary fuzzy support vector machine inference model and fmGA for construction management [56].

During project feasibility studies, conceptual cost estimates and its impact on the final project success are important. This is because such estimates provide important information which can help in the evaluation of the project, drawing of the engineering designs, planning, and management of the budget cost. A hybrid evolutionary fuzzy hybrid neural network (EFHNN) model was proposed by [57] for the improvement of the precision of conceptual cost estimates. In this model, a hybrid neural network (HNN), is first formed by combining neural networks (NN) with high-order NN (HONN). The next step is integration of fuzzy logic (FL) into the HNN to take care of the uncertainties; this gives rise to a fuzzy hybrid neural network (FHNN). Since a genetic algorithm is used in the FL and HNN to optimize the hybrid FHNN, the final version deployed in this work may be rightly called an 'EFHNN'. In this study, we calculated and compared the estimates of the overall and category costs for actual projects. From the results, the proposed EFHNN can be used as an accurate cost estimator at the initial phase of projects. However, the linear and non-linear neuron layer connectors in the EFHNN performed better than models that use a singular linear NN.

Project managers use EAC to project the total cost of a project at its completion because it is an important tool used to monitor the performance and risk of a project. High-level project decisions are often made by the executives, but they may lack the technical knowledge, and this may cause decision errors. The estimation of EAC using an evolutionary predictive model based on Gaussian process (GP) integrated with particle swarm optimization algorithm (PSO) has been proposed by [58]. The model proposed in this study was combined with an AI method and used to simulate human decision-making pattern in handling management-related problems. In this model, the input-output relationship was determined by the GP while PSO was used for the hyperparameters' optimization. The possibility of using the proposed hybrid model in EAC determination and in the calculation of the chances of a change in the predicted model monitor was investigated in this study. The model served as a reliable EAC estimator and can be of help to project managers when there is a need to improve project cost control effectiveness. The results achieved from the study were validated on real data from construction projects.

EVM is commonly used as an effective control method for the detection of project status and cost EAC prediction. Normally, the conventional EAC prediction methods extend the current project situation to the future by using a prior performance factor. The prediction of EAC and future event cost using a fuzzy neural network model has been performed by Feylizadeh et al. (2012) [59]. The fuzzy approach combined both qualitative and quantitative factors that affect EAC prediction. The effectiveness of this model was investigated on a real-world case study and the preliminary outcome of the investigation was encouraging.

During the construction phase of projects, cost overruns are frequently encountered; hence, there is a need for a proactive way of project costs monitoring and detecting potential problems. EAC is used in construction management as an indicator for helping project managers identify potential challenges and develop necessary solutions. In this study, weighted SVM, fuzzy logic, \& fast messy genetic algorithm were used to handle the 
distinct characteristics in EAC prediction [26]. The features of time series data were addressed in this study by employing the weighted SVM model while the fuzzy logic model was developed for the enhancement of the model's capability in approximate reasoning and to handle the uncertainties in the prediction of EAC. From the simulation studies, the developed model was found to achieve a significant enhancement in EAC modeling.

Subjective judgments can be exploited in a rigorous and formal way using a Bayesian approach based on expert opinion; this can lead to an improved estimate at completion accuracy within the EVM framework. This model was exploited by Caron et al. (2013) [60], as a combination of experts' knowledge with project data records in a bid to create a future-oriented and more valuable support tool that allows forecasting process improvement. This has a direct influence on the associated improvement in the decision-making process with respect to the project control. The proposed model in this study is robust and can be applied in every phase of a project, particularly during the early phase of a project when data records are unreliable. The model also allows the estimation of the confidence interval that describes future scenarios likely to be faced in the project. The effectiveness and applicability of the Bayesian approach were demonstrated and certified in the oil and gas industry. The results of the study also highlighted the possibility of applying the same approach to other industries for better project control. To improve the input and output management strategy in the Bayesian model and make it friendlier, the model has been translated into a software package.

A new study was developed in 2017 which focused on the use of ANN to establish an automated method for the estimation of the duration of dam projects construction [61]. In the study, the existing literature was reviewed while expert interviews were conducted on the critical variables that influence dam projects execution. Several data were collected on Iranian dam projects and used for the development of 7 ANN models. The performance was assessed using different datasets. The performance was assessed in terms of the root mean square error and correlation values which served as indicators for models' validity and reliability. To give the stakeholders the opportunity to use the ANN method to estimate the duration of dam project completion, a webbased automated prototype of the model was developed and validated. For better visualization, Table 1 displayed the adopted research on the AI applications for EAC modeling.

Table 1. The reviewed studies on the implementation of AI models cost projects over the last 10 years.

\begin{tabular}{|c|c|}
\hline Ref. & Research conclusion \\
\hline$[62]$ & $\begin{array}{l}\text { ANN model was used in this study for project cost simulation with the aim of improving the EVM } \\
\text { system. The outcome of the study suggested the suitability of the intelligent model in minimizing the } \\
\text { project cost-related errors. }\end{array}$ \\
\hline [56] & $\begin{array}{l}\text { SVM was combined with fast messy genetic algorithm (SVM-FMGA) and used for monitoring of } \\
\text { construction processes. The model was validated and approved for building cost estimation compared } \\
\text { to conceptual cost estimation. }\end{array}$ \\
\hline [57] & $\begin{array}{l}\text { The use of evolutionary fuzzy hybrid neural network for project cost estimation in industrial projects } \\
\text { was evaluated in this study. The outcome of the study showed that the approach is an optimistic way } \\
\text { of accurate cost estimation at the early project stages. }\end{array}$ \\
\hline [63] & $\begin{array}{l}\text { EAC prediction using an independent intelligent-based weighted SVM and fuzzy logic set was } \\
\text { presented in this study. The study relied on the fuzzy model to address the issue of uncertainty in the } \\
\text { time series data. }\end{array}$ \\
\hline [59] & $\begin{array}{l}\text { EAC estimation using a fuzzy neural network was presented here. The modelling was conducted in } \\
\text { consideration of various factors that affects the value of EAC. From the results, the approach is } \\
\text { considered good for EAC prediction from both the managers and contractors' perspectives. }\end{array}$ \\
\hline [60] & $\begin{array}{l}\text { A relatively new model for EAC computation was developed based on the integration of Bayesian } \\
\text { theory with the EVM framework. The applicability of the new model on EAC modelling was } \\
\text { validated. }\end{array}$ \\
\hline [30] & $\begin{array}{l}\text { A new EAC prediction method was presented via combination of the CEAC method with } 4 \text { growth } \\
\text { models; the study showed that the Gompertz model based EAC formula performed better than the } \\
\text { other indexed formulas. }\end{array}$ \\
\hline [64] & $\begin{array}{l}\text { The SVR model was analyzed to for EVM estimation and from the outcome of the analysis, the model } \\
\text { performed better than some of the available best-performing EVM methods on the same training data } \\
\text { set. }\end{array}$ \\
\hline [61] & $\begin{array}{l}\text { An ANN-based automotive programming approach was proposed for EAC prediction for a dam } \\
\text { construction project. The study showed that the developed method performed remarkably on the } \\
\text { investigated case study. }\end{array}$ \\
\hline [65] & $\begin{array}{l}\text { The genetic algorithm was integrated with deep learning prediction model for the estimation of the } \\
\text { EAC for residential fifteen projects. The research results confirmed the potential of the adopted } \\
\text { integrative prediction model and the feasibility of the deep learning over the literature models. }\end{array}$ \\
\hline
\end{tabular}




\section{Literature review assessment}

A mathematical or independent EAC estimate is often prepared (as a way of cross-checking the EAC) based on the cost and schedule experience using current performance indices. For instance, EAC can be computed using the CPI and Cumulative Budgeted Cost for Work Performed (ACWP) simply by using the CPI to divide the project BAC. The type of EAC resulting from such computation is often called Independent EAC (IEAC) and is different from the formal or grassroots EAC as it can be easily prepared and used for the validation of the possibility of using the current cost estimate and to notify when to undertake a comprehensive EAC. Note that these computations have no consideration for anticipated labour rate, efficiency, risk and opportunities, SRA, etc; hence, the are normally considered independent of sanity, logic and judgment but their computation is based on comparative analysis.

It is becoming an industrial practice to regularly prepare EACs along with the computation of the Best Case, Worst Case, and Most Likely EACs. At least, a comprehensive EAC (a complete "bottoms-up" EAC) is annually required on projects that are subject to the requirements of the Defence Federal Acquisition Regulation DFAR 252.234-7002 Earned Value Management System. A comprehensive EAC is also often prepared before initializing any major project as it can reflect the reduced uncertainty associated with the release of the project design and bill of material. The comprehensive EAC helps the project manager to provide the answer to the following questions:

i. Can the remaining authorized funds be enough to complete the project?

ii. Can the future cost performance be predicted from the previous cost experience?

iii. Is it possible to modify the remaining project based on the performance to date?

iv. Does the project cost performance have any impact on the corporate financial condition?

Therefore, the EAC and ECD must be released on time because both requires a sustained comparison with the project targets for the actual financial performance prediction for both the customers and the stockholders. Numerous business activities are nowadays managed as projects, and this has recently generated much interest in project management techniques which are developed for better project cost prediction and management. The first studies on project management-related studies were reported in the early 1950s with the intention of helping project managers in the identification of the critical project paths. Later, more researches were done to accommodate project risk management $\&$ analysis. It became obvious that consideration of tasks as given and predetermined would lead to erroneous outcomes in project management practice. The definition of a good schedule in real projects is often inadequate based on the deterministic processing times as these times are mere estimates that can change with time.

Project risks are outcomes of the uncertainty associated to an extent in all projects. Several works have been reported on the concept and measure of uncertainty. Projects are subject to some degrees of uncertainty due to several possible sources, such as resource unavailability, delays in the duration of activities, introduction of new project activities, deletion of some project activities, etc. However, the most notable source of uncertainty is the estimate of possible variability of the duration of project activity. Considering this stance, our choice involves modelling the times of processing project activities as random variables. Note that sometimes, the type of encountered uncertainty in certain projects may not conform with the axiomatic basis of probability theory and in such cases, probability-based approaches are ideal to be used, and have been applied in project management for many years.

Considering the limitations of the available EAC simulation methods, it has become important to develop a fast and reliable system that will address these cost control-related issues during project execution; hence, AIbased EAC prediction has been always the motive by construction management engineers and designers with the intention of addressing all the identified project cost management-related problems. Recently, the application of deep learning (DL) revealed a robust intelligent that can overcome the addressed limitation of the classical AI models.

\section{Possible future research}

The current review was highlighted the main formula, regression models and soft computing models introduced for the EAC calculation. It is worth to close this mini review article with some recommendation for future research. It is true that empirical formulas are associated with some limitations and time-consuming calculation; however, it is important when building intelligence model like artificial intelligence models to be aware of the critical predictors that have been stated in the empirical formulas to have more likely related model development to the actual status. Of recent discovered AI model, deep learning has emerged as one of the most attractive fields of science due to the limitation of the existing AI models and the notable theoretical and technological improvements [66]-[68]. Hence, deep learning has found application in several areas, such as self-driving cars, image recognition, text translation, etc. As a subfield of machine learning, deep learning is concerned with building algorithms that can learn and explain a high and low level of data abstraction which cannot be 
performed by the traditional ML algorithms. Deep learning models are often inspired by many knowledge sources, such as game theory and neuroscience; most of them also mimic the structure and function of the human nervous system. With the advancements in the field, scholars have begun to foresee a world where software can be coded with ease and allow for a more robust and generalized ways of solving problems [69].

Further, future studies trend should be implemented as a real-time application on other construction projects for a practical recognition of its contribution [70], [71]. It is worth to highlight, the current investigation was experimentally developed using historical information of residential construction projects. Thus, investigating other construction projects such as industrial can be an excellent example to be examined. This can aid in better monitoring of the project cycle and status. Another important associated drawback is the uncertainties of data, models and input parameters that can be checked in future study.

Conflicts of Interest: The authors declare no conflict of interest.

\section{References}

[1] S. Mohamed, "Safety climate in construction site environments," J. Constr. Eng. Manag., vol. 128, no. 5, pp. 375384, 2002.

[2] Z. M. Yaseen, Z. H. Ali, S. Q. Salih, and N. Al-Ansari, "Prediction of risk delay in construction projects using a hybrid artificial intelligence model," Sustainability, vol. 12, no. 4, p. 1514, 2020.

[3] J. Zeng, M. An, and N. J. Smith, "Application of a fuzzy based decision making methodology to construction project risk assessment," Int. J. Proj. Manag., vol. 25, no. 6, pp. 589-600, 2007.

[4] B. Dave and L. Koskela, "Collaborative knowledge management-A construction case study," Autom. Constr., vol. 18, no. 7, pp. 894-902, 2009.

[5] M. Martínez-Rojas, N. Marín, and M. A. Vila, "The role of information technologies to address data handling in construction project management,” J. Comput. Civ. Eng., vol. 30, no. 4, p. 4015064, 2016.

[6] S. T. H. Mortaji, R. Noorossana, and M. Bagherpour, "Project completion time and cost prediction using change point analysis," J. Manag. Eng., vol. 31, no. 5, p. 4014086, 2015.

[7] M. Cheng, H. Peng, Y. Wu, and T. Chen, "Automation in Construction Estimate at Completion for construction projects using Evolutionary Support Vector Machine Inference Model,” Autom. Constr., vol. 19, no. 5, pp. 619629, 2010.

[8] S. Vandevoorde and M. Vanhoucke, "A comparison of different project duration forecasting methods using earned value metrics,” Int. J. Proj. Manag., vol. 24, no. 4, pp. 289-302, 2006.

[9] M. Plaza and O. Turetken, "A model-based DSS for integrating the impact of learning in project control," Decis. Support Syst., vol. 47, no. 4, pp. 488-499, 2009.

[10] T. Narbaev and A. De Marco, "Cost estimate at completion methods in construction projects," in 2011 Proceedings of the 2nd International Construction and Project Management Conference, 2011, pp. 32-36.

[11] D. S. Christensen, R. C. Antolini, and J. W. McKinney, "A Review of Estimate at Completion Research," J. Cost Anal., vol. 12, no. 1, pp. 41-62, 1995.

[12] D. S. Christensen, "Determining an accurate estimate at completion," Natl. Contract Manag. J., vol. 25, pp. 1725, 1993.

[13] E. Huang and S.-J. Chen, "Estimation of project completion time and factors analysis for concurrent engineering project management: a simulation approach," Concurr. Eng., vol. 14, no. 4, pp. 329-341, 2006.

[14] E. Plebankiewicz, M. Juszczyk, and J. Malara, "Estimation of task completion times with the use of the PERT method on the example of a real construction project," Arch. Civ. Eng., vol. 61, no. 3, pp. 51-62, 2015.

[15] M. Y. Cheng and Y. W. Wu, "Evolutionary support vector machine inference system for construction management," Autom. Constr., vol. 18, no. 5, pp. 597-604, 2009.

[16] Z. M. Yaseen, "An insight into machine learning models era in simulating soil, water bodies and adsorption heavy metals: Review, challenges and solutions," Chemosphere, p. 130126, 2021.

[17] W. Jing, H. I. Naji, R. N. Zehawi, Z. H. Ali, N. Al-Ansari, and Z. M. Yaseen, "System Dynamics Modeling Strategy for Civil Construction Projects: The Concept of Successive Legislation Periods," Symmetry (Basel)., vol. 11, no. 5, p. 677, 2019.

[18] E. F. T. AlHares and C. Budayan, "Estimation at completion simulation using the potential of soft computing models: Case study of construction engineering projects," Symmetry (Basel)., vol. 11, no. 2, p. 190, 2019.

[19] I. Peško et al., "Estimation of costs and durations of construction of urban roads using ANN and SVM," Complexity, vol. 2017, 2017.

[20] A. Govindarajan, "A soft computing framework to evaluate the efficacy of software project management," in 2015 IEEE 9th International Conference on Intelligent Systems and Control (ISCO), 2015, pp. 1-6.

[21] A. Bermudez Peña, J. A. Lugo García, and P. Y. Piñero Pérez, "An adaptive-network-based fuzzy inference system for project evaluation,” Ing. y Univ., vol. 19, no. 2, pp. 299-313, 2015.

[22] M. Y. Cheng, H. C. Tsai, and C. L. Liu, "Artificial intelligence approaches to achieve strategic control over project 
cash flows," Autom. Constr., vol. 18, no. 4, pp. 386-393, 2009.

[23] C. M. Popescu and C. Charoenngam, Project planning, scheduling, and control in construction: An encyclopedia of terms and applications. John wiley \& Sons, 1995.

[24] J. Pajares and A. López-Paredes, "An extension of the EVM analysis for project monitoring: The Cost Control Index and the Schedule Control Index," Int. J. Proj. Manag., 2011.

[25] M.-Y. Cheng, H.-C. Tsai, C.-H. Ko, and W.-T. Chang, "Evolutionary fuzzy neural inference system for decision making in geotechnical engineering," J. Comput. Civ. Eng., vol. 22, no. 4, pp. 272-280, 2008.

[26] M. Cheng, N. Hoang, A. F. V Roy, and Y. Wu, "A novel time-depended evolutionary fuzzy SVM inference model for estimating construction project at completion," Eng. Appl. Artif. Intell., vol. 25, no. 4, pp. 744-752, 2012.

[27] D. S. Christensen, "Project advocacy and the estimate at completion problem," J. Cost Anal. Manag., vol. 1996, no. 2, pp. 35-60, 1996.

[28] D. S. Christensen, "Using performance indices to evaluate the estimate at completion," J. Cost Anal. Manag., vol. 1994, no. 2, pp. 17-24, 1994.

[29] F. T. Anbari, "Earned value project management method and extensions," Proj. Manag. J., vol. 34, no. 4, pp. 12 23, 2003.

[30] T. Narbaev and A. De Marco, "An Earned Schedule-based regression model to improve cost estimate at completion,” Int. J. Proj. Manag., vol. 32, no. 6, pp. 1007-1018, 2014.

[31] Q. W. Fleming and J. M. Koppelman, “Earned Value Project Management,” Engineering, vol. 16, pp. 19-23, 1998.

[32] T. Narbaev and A. De Marco, "Combination of Growth Model and Earned Schedule to Forecast Project Cost at Completion,” J. Constr. Eng. Manag., vol. 140, no. 1, p. 04013038, 2014.

[33] E. Kim, J. W. G. Wells, and M. R. Duffey, "A model for effective implementation of Earned Value Management methodology," Int. J. Proj. Manag., vol. 21, pp. 375-382, 2003.

[34] B. Kim and K. F. Reinschmidt, "Probabilistic Forecasting of Project Duration Using Bayesian Inference and the Beta Distribution,” J. Constr. Eng. Manag., no. March, pp. 178-186, 2009.

[35] M. Vanhoucke, Measuring time: Improving project performance using earned value management. Springer Science $\backslash \&$ Business Media, 2009.

[36] I. Henriques and P. Sadorsky, "The determinants of an environmentally responsive firm: An empirical approach," J. Environ. Econ. Manage., vol. 30, no. 3, pp. 381-395, 1996.

[37] R. Jakowski, "Duplication of colon in a Labrador Retriever with abnormal spinal column," Vet. Pathol., vol. 14, no. 3, pp. 256-260, 1977.

[38] J. L. Lollar, "Cost Performance Analysis Program for Use on Hand-Held Programmable Calculators," 1980.

[39] C. W. Parker, "C/SCSC and C/SSR Cost Performance Analysis Programs," DCAS Resid. Harris, DCASMS, Orlando, Florida, vol. 20, 1980.

[40] J. A. Totaro, “A Logical Approach to Estimate at Completion Formulas,” Progr. Manag., vol. 16, pp. $29-33,1987$.

[41] J. J. Haydon and R. O. Reither, "Methods of Estimating Contract Cost at Completion," ManTech Int. Corp. Virginia, vol. 31, 1982.

[42] J. Covach, J. J. Haydon, and R. O. Reither, "A Study to Determine Indicators and Methods to Compute Estimate at Completion (EAC)," Virginia ManTech Int. Corp., vol. 30, 1981.

[43] A. L. Blythe, "A Stochastic Model for Estimating Total Program Cost," ASD Reserv. Report, Aeronaut. Syst. Div. Wright-Patterson AFB, Ohio, 1982.

[44] J. M. Cryer and L. R. Balthazor, "Evaluation of Weighted Indices on Algorithms Utilized for Calculating Independent Estimates at Completion." ASD/HR MR Project, 1986.

[45] J. T. Sincavage, "TSARISM Time Series Analysis for Army Internal Systems Management," Army Aviat. Syst. Command, vol. 3, 1974.

[46] D. Olsen and R. W. Ellsworth, "Forecasting Techniques Employed in a Line Organization,” 1976.

[47] D. E. Busse, "A Cost Performance Forecasting Model," Air University, Maxwell AFB, Alabama, 1977.

[48] O. Karsch, "A cost performance forecasting concept and model," 1974.

[49] W. J. Weida, “A General Technique for R\&D Cost Forecasting,” 1977.

[50] G. K. Chacko, "Improving Cost and Schedule Controls Through Adaptive Forecasting," Concepts J. Def. Syst. Acquis. Manag., vol. 4, pp. 73-96, 1981.

[51] H. Watkins III, “An application of Rayleigh curve theory to contract cost estimation and control,” 1982.

[52] Z. El-Sabban, "Forecast of Cost/Schedule Status Utilizing Cost Performance Reports of the Cost/Schedule Control Systems Criteria: A Bayesian Approach.," US Army Aviat. Syst. Command. St. Louis Missouri, AD-754576, 1973.

[53] R. A. Hayes, "An Evaluation of a Bayesian Approach to Compute Estimates at Completion for Weapon System Programs," School of Systems and Logistics, Air Force Institute of Technology (AU), 1977.

[54] J. Holeman Jr, "A Product Improved Method for Developing a Program Management Office Estimated Cost at Completion," Virginia, 1975.

[55] M. Y. Cheng, H. C. Tsai, and W. S. Hsieh, "Web-based conceptual cost estimates for construction projects using Evolutionary Fuzzy Neural Inference Model,” Autom. Constr., vol. 18, no. 2, pp. 164-172, 2009. 
[56] M. Y. Cheng and A. F. V. Roy, "Evolutionary fuzzy decision model for construction management using support vector machine,” Expert Syst. Appl., vol. 37, no. 8, pp. 6061-6069, 2010.

[57] M.-Y. Cheng, H.-C. Tsai, and E. Sudjono, "Conceptual cost estimates using evolutionary fuzzy hybrid neural network for projects in construction industry," Expert Syst. Appl., vol. 37, no. 6, pp. 4224-4231, 2010.

[58] C.-C. Huang and M.-Y. Cheng, "Estimate at completion for construction projects Using Evolutionary Gaussian Process Inference Model," in International Conference on Multimedia Technology, 2011, pp. 4414-4417.

[59] M. R. Feylizadeh, A. Hendalianpour, and M. Bagherpour, "A fuzzy neural network to estimate at completion costs of construction projects," Int. J. Ind. Eng. Comput., vol. 3, no. 3, pp. 477-484, 2012.

[60] F. Caron, F. Ruggeri, and A. Merli, "A bayesian approach to improve estimate at completion in earned value management," Proj. Manag. J., vol. 44, no. 1, pp. 3-16, 2013.

[61] H. Golizadeh, S. Banihashemi, A. N. Sadeghifam, and C. Preece, "Automated estimation of completion time for dam projects,” Int. J. Constr. Manag., vol. 17, no. 3, pp. 197-209, 2017.

[62] S. H. Iranmanesh and M. Zarezadeh, "Application of artificial neural network to forecast actual cost of a project to improve earned value management system," in World Congress on Science, Engineering and Technology, 2008, pp. 240-243.

[63] M. Y. Cheng, N. D. Hoang, A. F. V. Roy, and Y. W. Wu, "A novel time-depended evolutionary fuzzy SVM inference model for estimating construction project at completion," Eng. Appl. Artif. Intell., vol. 25, no. 4, pp. 744752, 2012.

[64] M. Wauters and M. Vanhoucke, "Support Vector Machine Regression for project control forecasting," Autom. Constr., vol. 47, pp. 92-106, 2014.

[65] K. R. Kareem Kamoona and C. Budayan, "Implementation of Genetic Algorithm Integrated with the Deep Neural Network for Estimating at Completion Simulation,” Adv. Civ. Eng., vol. 2019, no. May, pp. 1-15, 2019.

[66] H. Liu, Y. He, Q. Hu, J. Guo, and L. Luo, "Risk management system and intelligent decision-making for prefabricated building project under deep learning modified teaching-learning-based optimization," PLoS One, vol. 15, no. 7, p. e0235980, 2020.

[67] S. Liu and W. Hao, "Forecasting the scheduling issues in engineering project management: Applications of deep learning models," Futur. Gener. Comput. Syst., vol. 123, pp. 85-93, 2021.

[68] R. Alkhaddar, T. Wooder, B. Sertyesilisik, and A. Tunstall, "Deep learning approach's effectiveness on sustainability improvement in the UK construction industry," Manag. Environ. Qual. An Int. J., 2012.

[69] Y. Lecun, Y. Bengio, and G. Hinton, "Deep learning," Nature. 2015.

[70] A. Gondia, A. Siam, W. El-Dakhakhni, and A. H. Nassar, "Machine Learning Algorithms for Construction Projects Delay Risk Prediction,” J. Constr. Eng. Manag., vol. 146, no. 1, pp. 1-16, 2020.

[71] M. N. Mahdi et al., "Software Project Management Using Machine Learning Technique—A Review," Appl. Sci., vol. 11, no. 11, p. 5183, 2021. 